\title{
Optimal Time Delay in the Control of Epidemic
}

\author{
Zhenggang Wang ${ }^{1}, \mathrm{KY}$. Szeto ${ }^{1 *}$, and Frederick Chi-Ching Leung ${ }^{2}$ \\ ${ }^{1}$ Department of Physics, Hong Kong University of Science and Technology, Clear Water \\ Bay, Hong Kong SAR \\ ${ }^{2}$ Department of Zoology, University of Hong Kong, Pokfulam Road, Hong Kong SAR, \\ People's Republic of China; \\ * Corresponding author: phszeto@ust.hk
}

\begin{abstract}
A mathematical model to address the efficiency of the isolation and quarantine strategies in the containment of epidemics is constructed based on the SIR model with time delay. The model is investigated with numerical simulation that demonstrates the importance of quick measure in identifying the infected and the subsequent quarantine of his/her neighbors. The model also provides a theoretical framework for the estimation of the cost involved in the containment of the epidemics. Based on a general estimate of the cost, we demonstrate the procedure for the calculation of the optimal set of parameters in our isolation and quarantine strategy through numerical simulation on a model social network. We find an important parameter $\pi$ which is a combination of several general parameters for the SIR model so that when $\pi>0$, the isolation and quarantine strategy will fail to contain the outbreak. The procedure outlined provides some general guidance in the selection of strategies in the containment of real epidemics, where the balance between social cost and risk must be carefully handled.
\end{abstract}

\section{Introduction}

The serious threat posed by the avian influenza viruses (H5N1), which may be transmissible among human [1,2], calls for effective strategies to control the pandemic[3-5]. The costly lesson from the SARS outbreak suggests that the short time delay in the identification and the isolation of the infected and a suitable period of quarantine should be an effective way to contain the highly contagious viruses spreading at its initial stage, especially before effective vaccine is developed $[6]$.

In order to mitigate the spread of contagious viruses, isolation with quarantine is a common measure to separate the healthy from the infected and the exposed. 
The effectiveness of this measure is determined by the strength and the efficiency of the practice. Typically, the time taken to identify and to isolate those infected and the imposed quarantine period [6-9] are the two most commonly used parameters in the execution of the strategy of containment. One generally expects that a very conservative and strict quarantine strategy is likely to contain the spreading. However, in the process of the implementation, the issues involving ethical, legal and socioeconomic concern $[10,11]$ must be properly addressed to avoid excessive measures in the isolation and quarantine at an unacceptable social cost. Here we seek a general model to describe the mathematical consequence of slow identification of the infected (large time delay for isolation) and the short quarantine period, thereby deriving a general guideline for the calculation of the optimal time delay (not the shortest) and the proper quarantine period (not too long but sufficient to contain the epidemics). The social network is modeled by a random network for simplicity [12], In the random network, any two nodes are linked with a constant probability $p$ equal to the average degree over the number of nodes $(p=<k>/ N)$. However, the procedure outlined in our simulation can be applied to others networks, in particular the real social network if known. We show with an example the general methodology in the computation of the optimal set of parameters by balancing the cost and the effectiveness of quarantine.

\section{Methods}

To illustrate the process of computing the optimal quarantine period for control of the spreading process in the early stage, we perform numerical simulation on a random network in a small population, with $N=500$ individuals and the average number of contacts is 10 (average degree $=$ number of nearest neighbors: $\langle k\rangle=10$ ).

For the transmission by daily contact, we assume an infection rate $\xi=0.001$ per day. For the infected individual, we assume that the incubation period is one day and the infectious period is $T_{R}$ days.

In probability theory, the Poisson distribution is a discrete probability distribution that expresses the probability of a number of events occurring in a fixed period of time if these events occur with a known average rate and is independently of the time since the last event. We here assume that the Poisson distribution properly describes the distribution of infectious period $T_{R}$. Since the typical infectious period ranges from two to seven days, with average around four days [3], we set the parameter " $a$ " in the Poisson distribution to be four with time measured in day, 


$$
\begin{aligned}
p(T) & =\frac{e^{-a} a^{T}}{T !} \quad \text { with } \\
a & =\langle T\rangle=\sum_{T=0}^{+\infty} T p(T)=4
\end{aligned}
$$

To simplify our model further, we also assume that the distribution of the number of days $T_{I}$ used for the identification and isolation of the infected individual also follows the Poisson distribution with the same mean.

In our SIR model with time delay and quarantine period, we have the following dynamical equation for the number of susceptible $(S)$, the number of infected $(I)$ and the number of quarantined $(Q)$

$$
\begin{aligned}
\frac{d S(t)}{d t} & =-\xi I(t) S(t)+U(t)+\beta_{R} I(t) \\
\frac{d I(t)}{d t} & =\xi I(t) S(t)-\beta_{R} I(t)-\beta_{I}\left(1-\beta_{R}\right) I\left(t-T_{Q}\right) \\
\frac{d Q(t)}{d t} & =\beta_{I}\left(1-\beta_{R}\right) I\left(t-T_{Q}\right)-U(t)
\end{aligned}
$$

Here $S+I+Q=N$ and $U$ is the number of individuals released after quarantine. The rate parameter shown in Fig.1 are given by the Poisson distribution,

$$
\begin{aligned}
& \beta_{R} \equiv \sum_{T_{R}>0} \frac{p_{R}}{T_{R}} \\
& \beta_{I} \equiv \sum_{T_{I}>0} \frac{p_{I}}{T_{I}}
\end{aligned}
$$

In our simple model which focus on the effect of time delay, we do not have death and the recovered population is returned to the population of susceptible. Those individuals identified, isolated and quarantined at time $t$ is given by the population quarantined $T_{Q}$ days before, and contributes the first term $\beta_{I}\left(1-\beta_{R}\right) I\left(t-T_{Q}\right)$ in Eq. 2 for the rate of change of $Q$. Similar term enters into the equation for the rate of change of $I$. We introduce the term $U$ to account for the released population from the quarantine area, so that it enters into the rate of change of $S$ with a plus sign, and with a minus sign in the rate of change of $Q$. 
Using $S=N-(I+Q)$ and $r=\xi N$, we can rewrite the rate of change of $I$ when there is quarantine, as

$$
\begin{aligned}
\frac{d I(t)}{d t}= & {\left[r-\beta_{R}-\beta_{I}\left(1-\beta_{R}\right)\right] I(t) } \\
& -\xi I(t)(I(t)+Q(t)) \\
& +\beta_{I}\left(1-\beta_{R}\right)\left(I(t)-I\left(t-T_{Q}\right)\right)
\end{aligned} .
$$

In the first term on the right of Eq.(4), the square bracket is an important parameter which we denote as

$$
\pi=r-\beta_{R}-\beta_{I}+\beta_{I} \beta_{R}
$$

Now, let's discuss the condition when the outbreak can be contained by the intervention measures. When we can contain the outbreak,

$$
\frac{d I(t)}{d t} \leq 0 \text { and } I(t) \leq I\left(t-T_{Q}\right)
$$

are satisfied. The critical point is defined by

$$
\frac{d I(t)}{d t}=0 \text { and } I(t)=I\left(t-T_{Q}\right)
$$

so that the third term in Eq.(4) is canceled out. We can see that at the initial stage of the outbreak when the quarantine strategy is employed, both $I$ and $Q$ are very small. the dominant term in Eq.4 is the first term, which is first order in $I$. The second term, which is quadratic in $I$ can be ignored. Thus, we can use the coefficient parameter $\pi$ in the first term of Eq.(4) as an index to measure the efficiency of the identification and the isolation of the infected. For $\pi<0$, the isolation is efficient and the spreading of the virus can be controlled. For $\pi>0$, the strategy is unlikely to contain the spread, though this measure still contribute in the mitigation of the scale of the outbreak. 


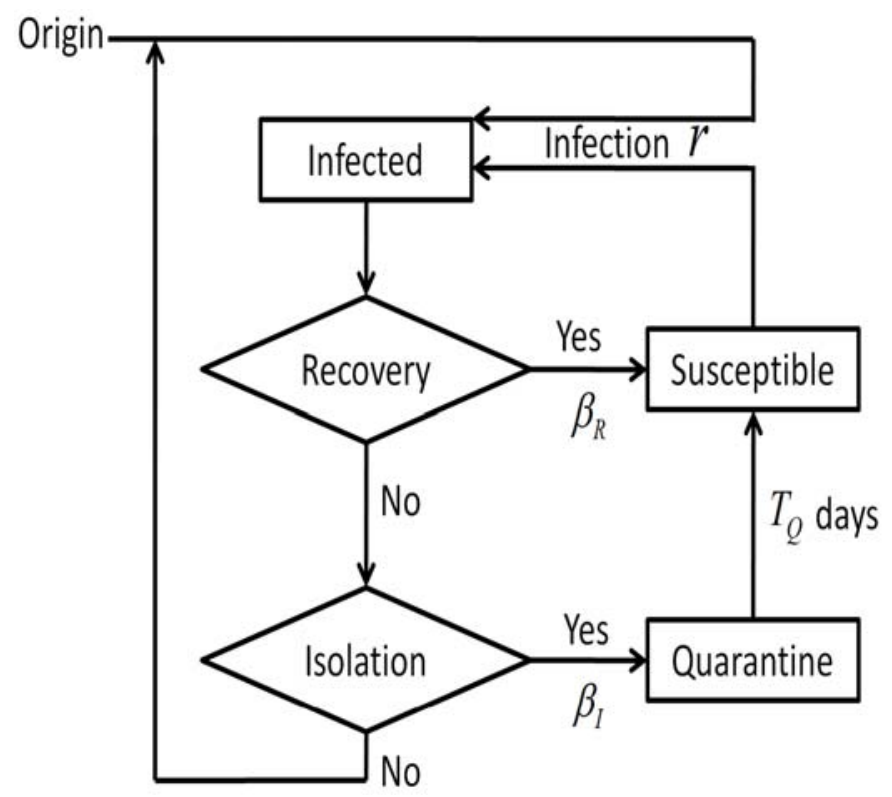

Fig.1. The flowchart of the numerical simulation performed in our model. The virus spreads with the infection rate, $r$. The infected individuals recover at the recovery rate $\beta_{R}$. After recovery, their status is susceptible. When the infected individual is identified and isolated at a rate $\beta_{I}$. After isolation, the neighbors of this infected individual are quarantined for $T_{Q}$ days.

\section{Results of Simulation}

Our simulations were performed according to the flowchart shown in Fig 1. Assuming that the virus is mildly pathogenic but highly contagious, we apply a quarantine strategy to control the outbreak. After a time delay $T_{I}$ an infected individual is identified and will be isolated and his/her nearest neighbors will also be quarantined and released after $T_{Q}$ days. We simulated the various outbreak patterns in the parameter space of $T_{I}$ and $T_{Q}$ to evaluate the effectiveness of differ- 
ent quarantine criteria. The data shown in Fig2b-d are averaged data over 500 simulations for each set of input parameters $\left(T_{I}, T_{Q}\right)$.
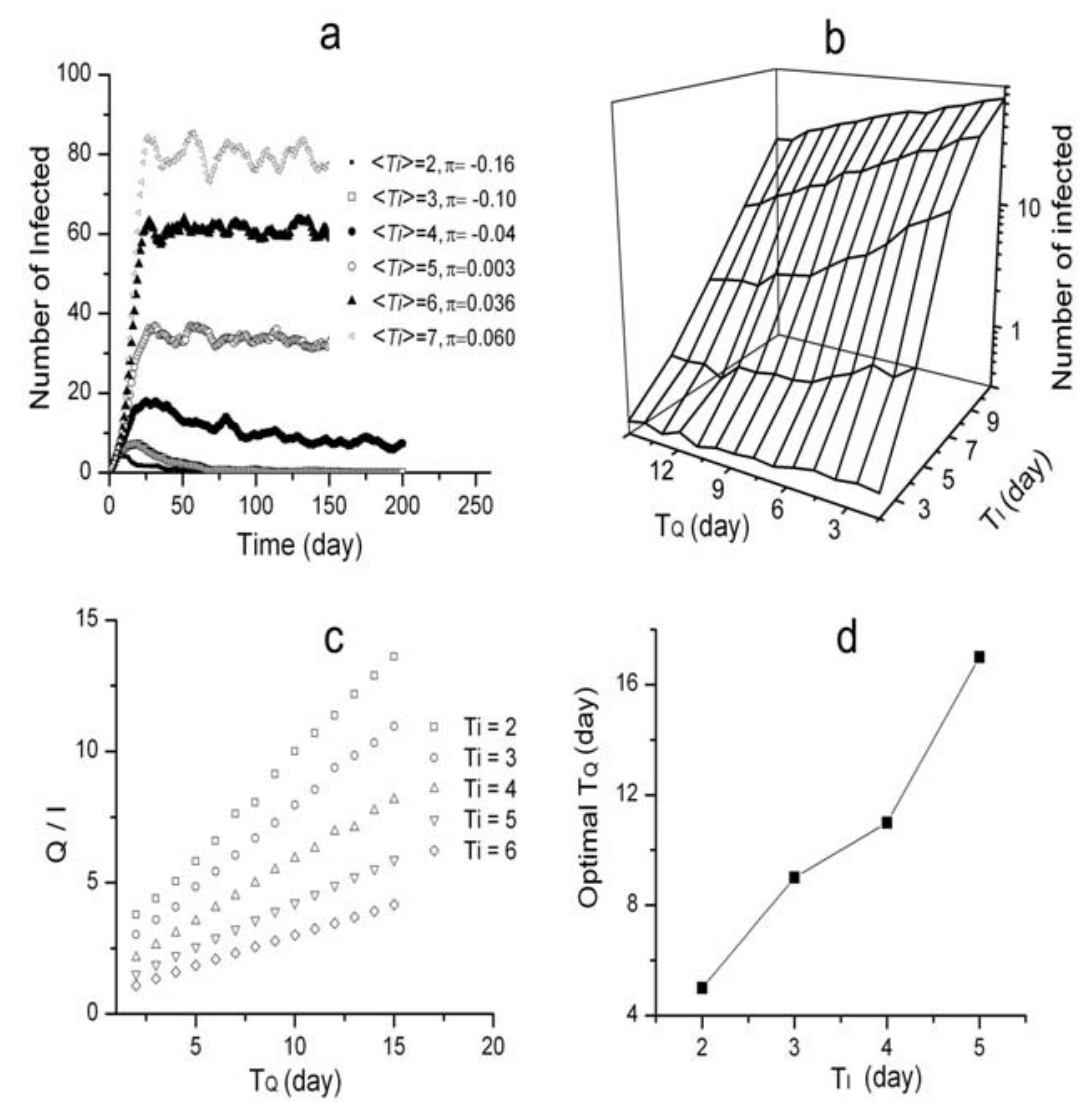

Fig.2. The simulations of the outbreak of influenza in a population with $\mathrm{N}=500$ individuals. (a). The infected number for different average time $T_{I}$ required to identify and then isolate the infected. The range of $T_{I}$ is $2,3,4,5,6,7$, corresponding to the set of values of $\beta_{I}=0.499,0.411,0.324,0.256,0.207,0.172$. When $\pi<0$, the outbreak is likely to be contained effectively with the quarantine strategy. (b) The average number of infected in the first 200 days with different $T_{I}$ and $T_{Q}$. (c) The ratio of the average number of quarantined and infected in the first 200 days with different $T_{I}$ and $T_{Q}$. (d) The optimal $T_{Q}$ for controlling the outbreak with minimum cost, assuming that the total cost for the strategy is proportional to the 
sum of the cost for the isolation of the infected and cost of the quarantining the neighbors.

The cost for the isolation of the infected is assumed to be twice the cost of the quarantine for his/her neighbors: total cost is proportional to $\mu I+Q$. In Fig.2a, when $T_{I}$ is small, ( $T_{I}=2,3$ days), the outbreak can be contained in the first 50 days, with the total number of infected minimized. However, when the time involved in identifying the infected, which is equivalent in our model to the time delay in the isolation of the infected goes above $4,\left(T_{I}>4\right)$, we have $\pi>0$ and the outbreak cannot be contained. If the quarantine period is constant, for example 4 days, the isolation measure will help to limit the transmission of virus and mitigate the outbreak. The more efficient the isolation, (in our model this is represented by a short time $T_{I}$ ), the smaller the scale of the outbreak. For a sufficiently short time delay in isolation $T_{I}$, we can make $\pi<0$, resulting in a complete containment of the outbreak.

The data shown in Fig $2 b, c$ and $d$ is the average data over 500 simulations for each set of input parameters $T_{I}$ and $T_{Q}$. In Fig $2 \mathrm{~b}$, we observe how the scale of the outbreak changes with different quarantine period. We see that generally the scale of the outbreak decreases with the quarantine period, which is common sense. Fig $2 \mathrm{c}$ shows that the ratio of the number of quarantined and infected steadily increases with the quarantined period, which means high percentage of healthy individuals are under quarantine, thus to strengthen the quarantine measure and mitigate the outbreak. This is quite reasonable. However, a long quarantine period may be too costly to be implemented. Therefore, we must investigate further the possibility of implementing a sufficiently long quarantine to minimize the scale of the outbreak, while satisfying the constraint that the cost is affordable.

The social cost is a complex function of various factors that involve not only the loss in monetary terms for the isolation and quarantine strategy, but also involve complex issues such as the loss of individual freedom of movement, interruption of important personal and public schedules, etc. A proper model for the cost of the strategy of isolation and quarantine is beyond the scope of our model, but still we can make a simple assumption that the social cost in the strategy is linearly proportional to the cost involving the infected (such as the cost for hospitalization) and the cost involving the quarantined (such as income lost and work delay). Also, we must remember that the cost changes for the viruses with different virulence. Thus, the first step in any control measure is to identify the virus. Now, let's assume that virus has been identified. We can then illustrate the method of computation as follow. If we assume that the ratio of the cost per infected individual over the cost per quarantined individual is $\mu$, then the social cost will be proportional to $\mu I+Q$, where $I$ is the number of infected individual and $Q$ is the number of individuals quarantined. Since both $I$ and $Q$ can be counted in our nu- 
merical simulation, we can estimate the social cost involved for given $\mu$, and the two parameters in our strategy: $T_{I}$ and $T_{Q}$. Thus, for each given $\mu$, we can obtain a three dimensional plot of social cost versus $T_{I}$ and $T_{Q}$. An example for the optimal quarantine period is shown in Fig. $2 \mathrm{~d}$ for $\mu=2$. The optimal quarantine period that achieves the minimum social cost (for $\mu=2$ ) can be numerically obtained for a given $T_{I}$. This optimal period $T_{Q}$ is shown in Fig.2d. Thus, for an assumed value of $\mu$ and time delay $T_{I}$, one can follow this procedure of estimating the optimal period for quarantine. Usually, the ratio $\mu$ is difficult to estimate and require more social studies. However, the time delay parameter $T_{I}$ can be readily obtained based on data of outbreaks in the past. Consequently, one can estimate the optimal period of quarantine as a function of social cost $\mu$ through our model, based on known input value of $T_{I}$.

\section{Discussion}

Our numerical simulation of a model based on SIR for the isolation and quarantine measure provides a quantitative evaluation for the traditional strategy in the control of the outbreak of infectious diseases. Since time is always needed to find the infected individual, there is always a time delay in his/her isolation. This time delay parameter, $T_{I}$, should be as short as possible in order to contain the outbreak at its initial stage. Nevertheless, $T_{I}$ can not be zero since instantaneous identification is technically impossible. We therefore incorporate this finite $T_{I}$ in our model and consider the effectiveness of various periods of quarantine in the containment of the outbreak. From our mathematical model, we find a critical parameter $\pi$ that provides an indicator to measure the efficiency of a quarantine policy in the limitation and containment of an outbreak. For $\pi>0$, the outbreak can not be contained with simple quarantine measure, though quarantine can still reduce the scale of the outbreak. For $\pi<0$, the measure is efficient and the outbreak can be contained.

Besides the introduction of the critical parameter $\pi$, we also investigate the optimal period of quarantine, taking into account of the social cost involved. In principle, an outbreak can always be controlled with a sufficiently long quarantine period. However, the social cost involved in a long quarantine period can be too high to be practical $[6,7,9-11]$. In order to balance the cost issue with an effective containment of the outbreak, we illustrate the procedure to calculate the optimal quarantine period that achieves the minimum social cost for a given $T_{I}$ through a simple example. When deciding on the proper measure for the control of the outbreak at its early stage, a policy maker must make a compromise between two 
competing factors: the cost of taking risk on public health and the social cost incurred by quarantine on the economy. Since the quarantine time is a critical factor in containing the outbreak at the initial stage, we here provides a practical procedure for its estimation.

In a more realistic application, one should extend the present analysis to a more detailed model of social network. One important variation is to consider isolation measure that takes into account of hubs or community structures in social network that may be the centers of outbreak. In this connection, the actual location where isolation and quarantine measures are applied is important, as recently discussed in ref [13]. The details of the dynamics process of virus spreading can also be further investigated in networks of various topologies using techniques in nonequilibrium statistical physics [14-16].

Acknowledgment K.Y. Szeto acknowledges the support of grant CERG602506 and 602507

\section{References}

1. Webster RG, Peiris $\mathrm{M}, \mathrm{Chen} \mathrm{H}$, et al. $\mathrm{H} 5 \mathrm{~N} 1$ outbreaks and enzootic influenza. Emerg Infect Dis 12:3-8 (2006)

2. Brankston G, Gitterman L, Hirji Z, et al. Transmission of influenza A in human beings. Lancet Infect Dis 7:257-65(2007)

3. Ferguson NM, Cummings DA, Cauchemez S, et al. Strategies for containing an emerging influenza pandemic in Southeast Asia. Nature 437:209-14(2005)

4. Ferguson NM, Cummings DA, Fraser C, et al. Strategies for mitigating an influenza pandemic. Nature 442:448-52(2006)

5. Longini IM, Jr., Nizam A, Xu S, et al. Containing pandemic influenza at the source. Science 309:1083-7(2006)

6. Moore J. Pandemic influenza and the law: isolation, quarantine, and other legal tools for containing outbreaks. N C Med J 68:59-61(2007)

7. DiGiovanni C, Bowen N, Ginsberg M, et al. Quarantine stressing voluntary compliance. Emerg Infect Dis 11:1778-9(2005)

8. Gostin LO. Influenza pandemic preparedness: legal and ethical dimensions. Hastings Cent Rep 34:10-1(2004)

9. Letts J. Ethical challenges in planning for an influenza pandemic. N S W Public Health Bull 17:131-4(2006)

10. Gostin LO, Bayer R, Fairchild AL. Ethical and legal challenges posed by severe acute respiratory syndrome: implications for the control of severe infectious disease threats. Jama 290:3229-37(2003)

11. Gostin LO, Gravely SD, Shakman S, et al. Quarantine: voluntary or not? J Law Med Ethics 32:83-6(2004)

12. Erdos P, Renyi A. On Random Graphs. Publicationes Mathematicae 1959;6:290297. 
13. Zhenggang Wang, Kwok Yip Szeto and Frederick Chi-Ching Leung; "Effectiveness of Closure of Public Places with Time Delay in Disease Control", Journal of Integrative Bioinformatics, 5(2):96(2008)

14. Z.Z. Guo and Kwok Yip Szeto; Survivor statistic and damage spreading on social network with power-law degree distributions; Physica A374(2007) 471-477

15. Z.Z. Guo and Kwok Yip Szeto, Damage spreading in two-dimensional trivalent cellular structures with competing Glauber and Kawasaki dynamics. Phys. Rev. E71, 066115(2005)

16. Z.Z.Guo, K.Y. Szeto, and Xiujun Fu, Damage spreading on two-dimensional trivalent structures with Glauber dynamics: Hierarchical and random lattices, Phys. Rev. E70, 016105(2004) 